\title{
Data-Driven Optimized Tracking Control Heuristic for MIMO Structures: A Balance System Case Study
}

\author{
Ning Wang, Mohammed Abouheaf and Wail Gueaieb
}

\begin{abstract}
A data-driven computational heuristic is proposed to control MIMO systems without prior knowledge of their dynamics. The heuristic is illustrated on a two-input two-output balance system. It integrates a self-adjusting nonlinear threshold accepting heuristic with a neural network to compromise between the desired transient and steady state characteristics of the system while optimizing a dynamic cost function. The heuristic decides on the control gains of multiple interacting PID control loops. The neural network is trained upon optimizing a weightedderivative like objective cost function. The performance of the developed mechanism is compared with another controller that employs a combined PID-Riccati approach. One of the salient features of the proposed control schemes is that they do not require prior knowledge of the system dynamics. However, they depend on a known region of stability for the control gains to be used as a search space by the optimization algorithm. The control mechanism is validated using different optimization criteria which address different design requirements.
\end{abstract}

Index Terms-Optimal Control, Nonlinear Control, Nonlinear Threshold Accepting Heuristic, Neural Networks

\section{INTRODUCTION}

The control problem of balance systems belongs to a class of nonlinear control systems which is usually solved using analytical as well as numerical techniques [1]-[3]. The goal of this work is to develop a position-angle regulation scheme of an inverted-pendulum-cart system using two interacting PID control loops. The PID control gains are decided using a nonlinear threshold accepting heuristic. Additional neural network state feedback mechanism is employed to optimize the total dynamic cost during the regulation processes. Elmer Sperry introduced a PID scheme in 1911 in order to solve the steering problem of an automatic ship and Nicholas Minorsky designed another type in 1922 [4]. The PID mechanisms are used in applications like manipulator control in robotic arms [5], control of unmanned aerial vehicles [6], industrial hydraulic regulators [7], temperature control [8], etc.

Nonlinear Threshold Accepting (NLTA) heuristic is developed by Nahas and Nourelfath, and it relies on a nonlinear accepting threshold criterion formed using a low-pass filter scheme [9], [10]. The NLTA heuristic finds a solution for the optimization problem by continuously updating the local search outcome starting from a random initial feasible guess. The accepting rule employs a magnitude of a low pass filter

Ning Wang, Mohammed Abouheaf, and Wail Gueaieb, are with School of Electrical Engineering and Computer Science, University of Ottawa, Ottawa, Ontario, Canada. e-mail: \{nwang094,mabouhea,wgueaieb\}@uOttawa.ca transfer function. It searches for a better feasible neighboring solution as will be explained later on. This approach is used to solve many NP-hard problems in [9]. It tackled energy distribution optimization problems like power system dispatch with prohibition zones and multiple fuel options in [11]. NLTA is employed to find solutions for the redundancy allocation where it is applied to solve the redundancy allocation problems and enhance the associated reliability in [12]. Further, it is employed to regulate the load frequency and automatic voltage disturbances for a network of power generation units in [13].

The Artificial Neural Network (ANN) is a class of the artificial intelligence sciences, and it is widely used to solve the nonlinear optimization problems. They are employed in power systems to control electric loads where they outperformed other regression approaches [14]. Neural networks are used to implement solutions for a class of adaptive control problems in [15]. An adaptive neural network scheme is employed to design a nonlinear flight controller in [16]. A data-driven method for computing reachable sets is used to estimate the attractions domains of model predictive controllers in [17]. A dynamic-event triggering control strategy based on integral reinforcement learning is proposed for partially unknown nonlinear systems in [18]. In [19], a fuzzy-neural network approach is employed to control a a flexible wing aircraft.

The work is organized as follows; Section II explains the dynamical model of a balance system. The development of PID angle-position control loops are detailed out in Section III. Further, a nonlinear state feedback control mechanism is realized using a neural network in Section IV. The usefulness and analysis of the presented schemes are shown in Section V. Finally, concluding remarks are pointed out in Section VI.

\section{Dynamics of a Balance System}

The dynamics of a balance system along with the objectives of the control problem are presented in this section.

\section{A. Inverted-Pendulum-Cart Dynamics}

A free-body-sketch of an inverted-pendulum-cart system is shown in Fig. 1 [20]. The pendulum swings as the moving cart slides along the $x$-direction due to a horizontal force $u$. The masses of a point rigidly connected at the top of the pendulum and the cart are denoted by $m$ and $M$, respectively. The parameters $l$ and $x$ refer to the pendulum's length and displacement of the cart, respectively. The angle $\theta$ is spanned, 
from the upright reference, by the pendulum. The dynamics of the balance system are arranged as follows [20]:

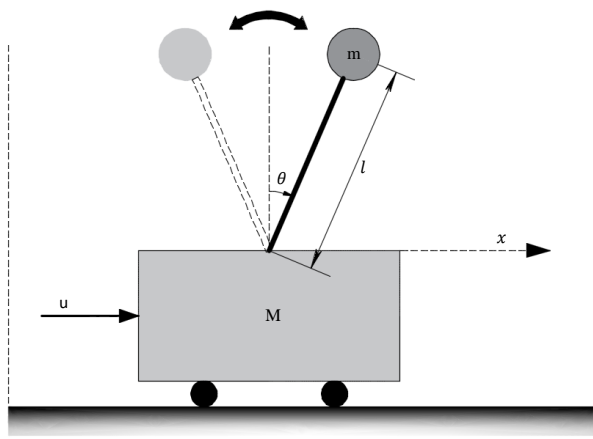

Fig. 1. Free-body diagram for a balance system.

Firstly, the ball-coordinates $\left(x_{p}, y_{p}\right)$ refer to the center of gravity of the inverted pendulum and they are given by $x_{p}=x+l \sin \theta$ and $y_{p}=l \cos \theta$. The force balance in the $x$ direction is expressed as follows $M \frac{d^{2} x}{d t^{2}}+m \frac{d^{2} x_{p}}{d t^{2}}=u$. Then $(M+m) \ddot{x}-m l \sin \theta(\dot{\theta})^{2}+m l \cos \theta \ddot{\theta}=u$. Secondly, the force components of the inverted pendulum in the $x$ and $y$ directions are given by $F_{p x}=m \frac{d^{2} x_{p}}{d t^{2}}$ and $F_{p y}=m \frac{d^{2} y_{p}}{d t^{2}}$. The underlying torque equation is given by $\left(F_{p x} \cos \theta\right) l-\left(F_{p y} \sin \theta\right) l=(m g \sin \theta) l$, where $g$ is the gravitational acceleration. Then $m \ddot{x} \cos \theta+m l \ddot{\theta}=m g \sin \theta$. A state space representation can be obtained using means of Jacobian framework around equilibrium (i.e., $\theta=0$ ) so that

$$
\dot{X}=A X+B u
$$

where $X=\left[\begin{array}{c}\theta \\ \dot{\theta} \\ x \\ \dot{x}\end{array}\right], A=\left[\begin{array}{cccc}0 & 1 & 0 & 0 \\ \frac{(M+m) g}{M l} & 0 & 0 & 0 \\ 0 & 0 & 0 & 1 \\ \frac{-m g}{M} & 0 & 0 & 0\end{array}\right], B=\left[\begin{array}{c}0 \\ \frac{-1}{M l} \\ 0 \\ \frac{1}{M}\end{array}\right]$.

\section{B. Formulation of the Control Problem}

The goal of the optimization or control problem is to let the cart and pendulum follow the desired position-angle trajectories (i.e., $x^{r e f}$ and $\theta^{r e f}$ ) using two PID-control loops.

\section{Position-Angle Control Mechanism}

This section introduces a coupled position-angle PID control mechanism for the balance system using an NLTA approach.

\section{A. PID Control System}

The interacting PID control loops are shown in Fig. 2. It is required to drive the position and angle tracking errors (i.e., $e_{x}=x^{r e f}-x$ and $e_{\theta}=\theta^{r e f}-\theta$ ) to zeros, respectively. The underlying control signals $u_{P I D}^{x}$ and $u_{P I D}^{\theta}$, generated using the PID-control loops, are given by $u_{P I D}^{j}(t)=K_{p}^{j} e_{j}(t)+$ $K_{i}^{j} \int_{0}^{t} e_{j}(\eta) d \eta+K_{d}^{j} \frac{d e_{j}(t)}{d t}, j=\{x, \theta\}$ where $K_{p}^{j}, K_{i}^{j}, K_{d}^{j}$ are PID control gains. The aggregate input control signal is given by $u(t)=u_{P I D}(t)=u_{P I D}^{x}(t)+u_{P I D}^{\theta}(t)$.

As is evident from the dynamics of the balance system, the cart acceleration is coupled to that of the pendulum and vice versa. Therefore, the control gains of the two loops cannot be independently tuned. Instead, both control units are treated by the NLTA heuristic as a single controller with six parameters to tune (i.e., search for the tuple $\left(K_{p}^{x}, K_{i}^{x}, K_{d}^{x}, K_{p}^{\theta}, K_{i}^{\theta}, K_{d}^{\theta}\right)$ in a 6-dimensional search space).
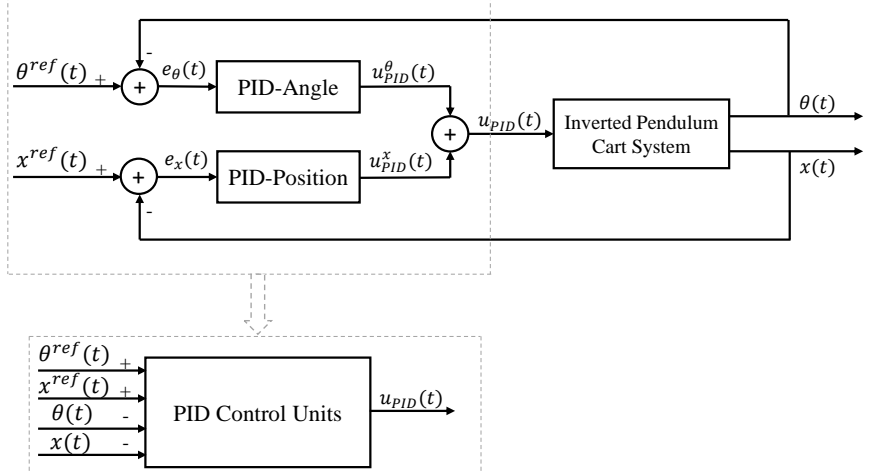

Fig. 2. Position-angle PID-control loops.

\section{B. NLTA-Based PID Gain Tuning}

The NLTA heuristic finds a solution based on a predefined objective function which reflects a specific desired performance. As a matter of fact, one can adopt a cost function to influence the system's transient and steady state characteristics. However, some of these objectives may be contradictory. For example, a shorter settling time may lead to a higher overshoot. Herein, we will suggest a number of cost functions to be adopted by the NLTA approach. Each one exploits a compromise between some of the system's response characteristics.

The first optimization criterion we consider is a convex cost function that minimizes the Integrated Squared Errors (ISE) given by ISE $=\int_{0}^{t}\left(w_{\theta} e_{\theta}^{2}(\eta)+w_{x} e_{x}^{2}(\eta)\right) d \eta$, where $w_{\theta}$ and $w_{x}$ are some weighting constants. In this case, we took $w_{\theta}=w_{x}=0.5$. Another objective function is considered to reduce the overshoot along with the ISE. We call it the "ISE and Absolute Error" criterion (ISE-AB). It is defined as $\mathrm{ISE}-\mathrm{AB}=\int_{0}^{t}\left(w_{\theta} e_{\theta}^{2}(\eta)+w_{x} e_{x}^{2}(\eta)+w_{\theta}^{\prime}\left|e_{\theta \mid(\eta)}+w_{x}^{\prime}\right| e_{x}(\eta) \mid\right) d \eta$, where $w_{\theta}, w_{x}, w_{\theta}^{\prime}$, and $w_{x}^{\prime}$, are weight constants, which in this work are initialized to $w_{\theta}=w_{x}=w_{\theta}^{\prime}=w_{x}^{\prime}=0.25$. The third objective function tackles the cart response settling time $T_{s}$ along with the ISE. We call it the "ISE and Settling Time" criterion (ISE-TS) and define it by ISE-ST = $\int_{0}^{t}\left(w_{\theta} e_{\theta}^{2}(\eta)+w_{x} e_{x}^{2}(\eta)\right) d \eta+w_{s} T_{s}$, where $w_{s}$ is the weight associated to the settling time. Here, the weights are set as $w_{\theta}=w_{x}=0.5$ and $w_{s}=0.1$. The final objective function addresses the ISE and the cart response overshoot. It is referred to as the "ISE and OverShoot" criterion (ISE-OS) and it is expressed as ISE-OS $=\int_{0}^{t}\left(w_{\theta} e_{\theta}^{2}(\eta)+w_{x} e_{x}^{2}(\eta)\right) d \eta+w_{o}$ OS, where $w_{o}$ is the weight associated to the overshoot. We fixed the weights to $w_{\theta}=w_{x}=0.5$ and $w_{o}=0.1$. The optimization process using the NLTA approach is detailed out in Algorithm 1. 


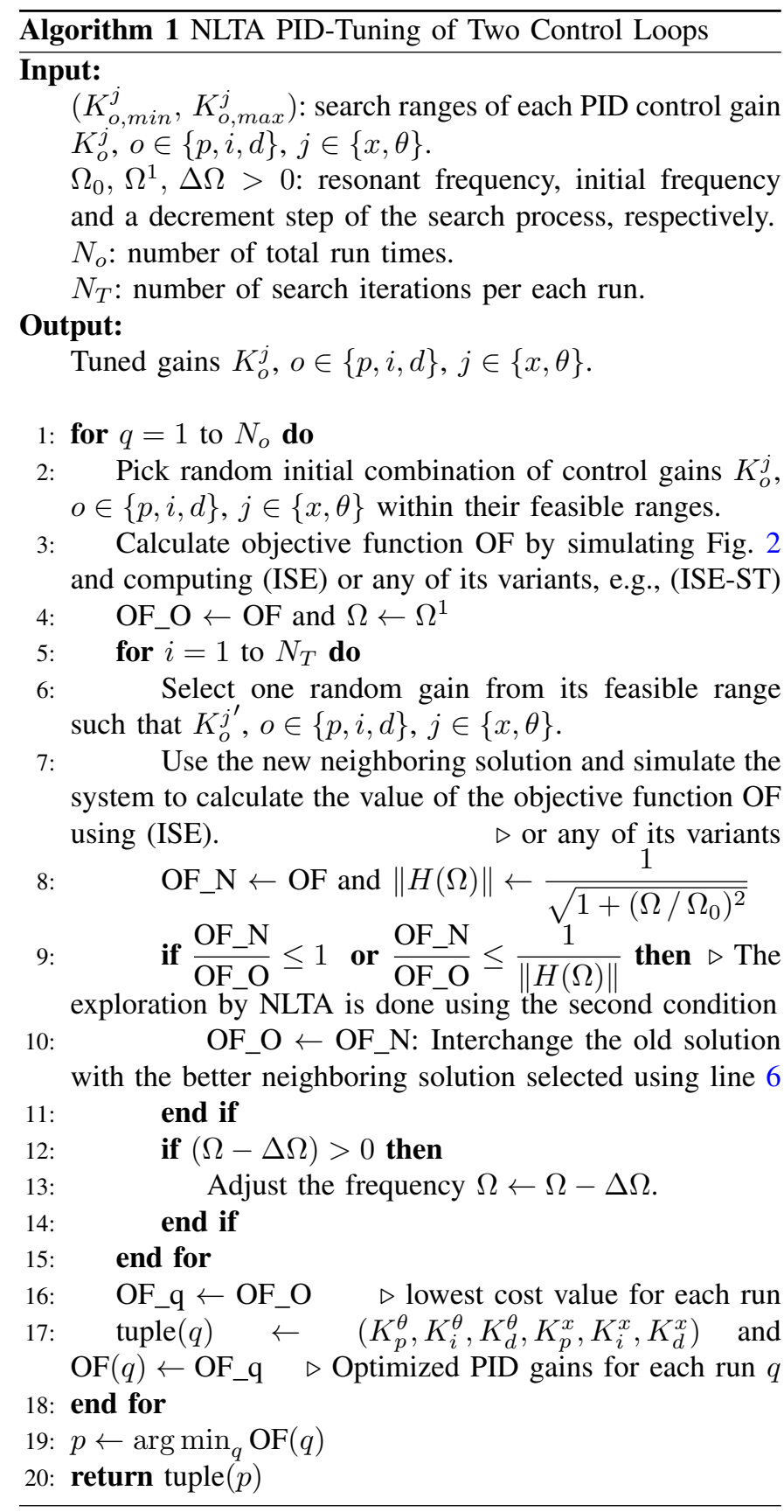

\section{State Feedback Control Mechanism}

The control interest is not only to regulate the referencetracking errors but also to optimize a broader objective function that may encompass other signals as well. In the sequel, a state feedback mechanism based on a neural network is developed and then compared to another controller that is based on solving the system's Algebraic Riccati Equation (ARE) [20].

\section{A. Neural Network Optimization Algorithm}

A feedforward NN is trained to optimize a total dynamical cost of the balance system. The overall control scheme including the full state neural network optimization loop and the PID control loops is shown in Fig. 3 (i.e., $u=u_{P I D}+u_{N N}$ ).

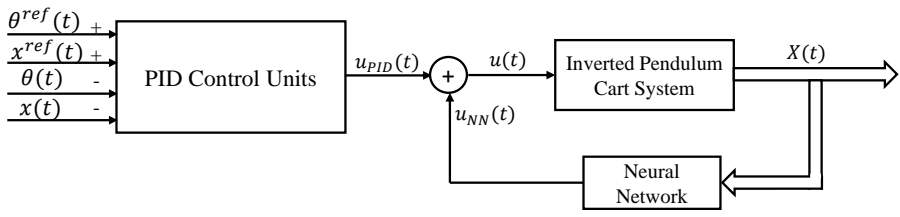

Fig. 3. Overall PID-Neural Network control scheme.

The training samples are prepared using a Q-Table process where discretized state-action combinations are employed [19]. Then, according to a performance criteria (i.e., an objective cost function), a suitable control signal is decided. The objective criterion adopted herein is given as follows

$$
F_{k}=X_{k+1}^{T} Q^{N N} X_{k+1}+R^{N N}\left(u_{k}^{N N}\right)^{2},
$$

where $k$ is a time-index, $Q^{N N} \geq 0 \in \mathbb{R}^{4 \times 4}$ and $R^{N N}>0 \in$ $\mathbb{R}$ are symmetric matrix and positive scalar value respectively. The rest of the details are illustrated in Algorithm 2 [19].

\section{B. Linear Quadratic Regulator}

The linear quadratic regulator (LQR) approach [21] provides an optimal solution to (1) while minimizing the quadratic performance index $J=\int_{0}^{\infty} U(X(\eta), u(\eta)) d \eta$, where $U=$ $\frac{1}{2}\left(X^{T}(\eta) Q X(\eta)+R u^{2}(\eta)\right), Q \geq 0$ and $R>0$ are weighting matrices, and $X$ is a state vector. The objective is to find the optimal control law $K$ where the optimal control signal is given by $u^{o}=-K X, K=R^{-1} B^{T} P$, where $P \geq 0 \in \mathbb{R}^{n \times n}$ is the solution for the ARE [21]. The LQR is integrated in the closed loop as shown in Fig. 4.

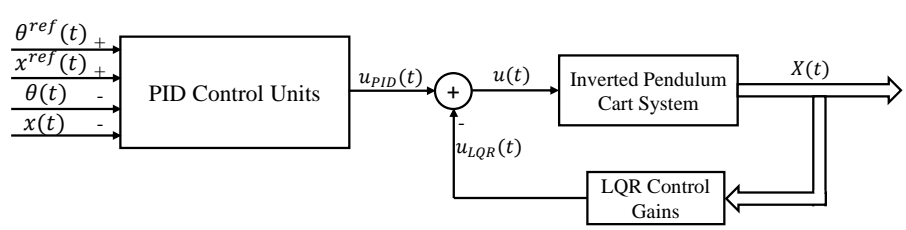

Fig. 4. Combined PID-LQR control scheme.

\section{Simulation Results}

The proposed control schemes are integrated together in a closed-loop structure with the balance system. The system's physical parameters are listed in Table I [20]. Hence, the state space matrices of the system are given by

$$
\begin{gathered}
A=\left[\begin{array}{cccc}
0 & 1 & 0 & 0 \\
29.8615 & 0 & 0 & 0 \\
0 & 0 & 0 & 1 \\
-0.9401 & 0 & 0 & 0
\end{array}\right], \quad B=\left[\begin{array}{c}
0 \\
-1.1574 \\
0 \\
0.4167
\end{array}\right], \quad X_{0}=\left[\begin{array}{l}
0 \\
0 \\
0 \\
0
\end{array}\right] . \\
\text { TABLE I } \\
\text { PARAMETERS OF THE INVERTED-PENDULUM-CART SYSTEM }
\end{gathered}
$$

\begin{tabular}{cccc}
\hline Parameter & Value & Parameter & Value \\
\hline$M$ & $2.4 \mathrm{~kg}$ & $m$ & $0.23 \mathrm{~kg}$ \\
$l$ & $0.36 \mathrm{~m}$ & $g$ & $9.8 \mathrm{~m} / \mathrm{s}^{2}$ \\
\hline
\end{tabular}

The cart and pole reference positions are taken as $x^{\text {ref }}(t)=$ $0.1 \mathrm{~m}$ and $\theta^{\text {ref }}(t)=0, \forall t \geq 0$. The simulations are conducted using Matlab-Simulink environment. 
Algorithm 2 Neural Network Energy Optimization Scheme Input:

$\left(X_{i_{\min }}, X_{i_{\max }}\right)$ : feasible range of each state $X_{i} \in X$, $i=1,2, \ldots, n$ with a discrete step $\Delta X_{i}$.

$\left(u_{\min }^{N N}, u_{\max }^{N N}\right)$ range of $u^{N N}$ with discrete-step $\Delta u^{N N}$.

Output:

Nonlinear State Feedback Neural Network Optimizer NN

1: The action space $\left[u_{\min }^{N N}, u_{\max }^{N N}\right]$ is discretized into $N_{u}$ discrete control values: $\left[u_{1}^{N N}, u_{2}^{N N}, \ldots, u_{N_{u}}^{N N}\right]$.

2: The state space is discretized so that $\left[X_{1_{\text {min }}}, X_{1_{\text {min }}}+\right.$ $\Delta X_{1}, X_{1_{\min }}+2 \Delta X_{1}, \ldots, X_{1_{\max }}, X_{2_{\text {min }}}, X_{2_{\text {min }}}+$ $\Delta X_{2}, X_{2_{\text {min }}}+2 \Delta X_{2}, \ldots, X_{2_{\max }}, \ldots, X_{n_{\min }}, X_{n_{\min }}+$ $\left.\Delta X_{n}, X_{n_{\min }}+2 \Delta X_{n}, \ldots, X_{n_{\max }}\right]$ and then tuples of all valid combinations of the different states are stored in a single row with $N_{X}$ entries.

3: Form a Q-Table $Q\left(1 \ldots N_{u}, 1 \ldots N_{X}\right)$, whose row and column indices refer to those of the discrete action and state spaces found as per lines 1 and 2, respectively.

4: Populate the Q-Table with the objective function (2). Then, decide the control action with the lowest value.

5: Train a single hidden layer feedforward NN using $N_{X}$ samples, each with $n$ inputs (combination $X_{1}, X_{2}, . ., X_{n}$ ) and one output (best control decision $u$ as per line 4).

6: return Trained Neural Network (NN).

\section{A. Performance Analysis of the Different PID Schemes}

At first, the system is simulated with only PID loops, as depicted in Fig. 2. The NLTA algorithm is applied offline to tune the gains of the PID controllers. The parameters adopted for the simulations are listed in Table II.

TABLE II

PARAMETERS OF THE NLTA OPTIMIZER

\begin{tabular}{cccc}
\hline Parameter & Value & Parameter & Value \\
\hline$\left(K_{p_{\text {min }}}^{\theta}, K_{p_{\max }}^{\theta}\right)$ & $(-44,-36)$ & $\left(K_{i_{\text {min }}}^{\theta}, K_{i_{\text {max }}}^{\theta}\right)$ & $(-2,2)$ \\
$\left(K_{d_{\min }}^{\theta}, K_{d_{\max }}^{\theta}\right)$ & $(-10,-6)$ & $\left(K_{p_{\min }}^{x}, K_{p_{\max }}^{x}\right)$ & $(-3,1)$ \\
$\left(K_{i_{\min }}^{x}, K_{i_{\max }}^{x}\right)$ & $(-2,2)$ & $\left(K_{d_{\min }}^{x}, K_{d_{\max }}^{x}\right)$ & $(-5,-1)$ \\
$\Omega_{0}, \Omega_{1}[\mathrm{rad} / \mathrm{s}]$ & 200,50 & $\Delta \Omega[\mathrm{rad} / \mathrm{s}]$ & 0.005 \\
N_T & 1000 & N_o & 10 \\
\hline
\end{tabular}

The optimization outcomes, after 10 search-runs, associated with the objective functions defined earlier are summarized in Table III. It is noticed that the specialized objective functions are successful in minimizing their target criteria. For example, the ISE-TS and ISE-OS criteria led to the best settling time and overshoot, respectively.

The PID gains obtained using the technique proposed in [20] and the NLTA approach, using the predefined objective functions, are listed in Table IV. The dynamic cost, transient and steady state characteristics are listed in Table V. It shows that, the four variants of the PID controllers optimized with the NLTA outperformed the method proposed in [20] in all aspects. Further, the last two columns of Table V assesses performance measures related to the LQR and the ANN optimizers. Although none of these optimizers are enabled yet
TABLE III

PID TUNING OUTCOMES WITH DIFFERENT COST FUNCTIONS

\begin{tabular}{llcc}
\hline $\begin{array}{l}\text { Objective } \\
\text { Function }\end{array}$ & $\begin{array}{l}\text { Optimization } \\
\text { Criterion }\end{array}$ & $\begin{array}{c}\text { Minimum } \\
\text { Value }\end{array}$ & $\begin{array}{c}\text { Maximum } \\
\text { Value }\end{array}$ \\
\hline ISE & Rise Time [s] & 0.8122 & 0.9149 \\
& Settling Time [s] & 3.3351 & 5.0759 \\
& Overshoot [\%] & 1.4904 & 6.7221 \\
& ISE & 0.4558 & 0.4658 \\
\hline ISE-AB & Rise Time [s] & 0.9532 & 1.3752 \\
& Settling Time [s] & 1.8481 & 3.6533 \\
& Overshoot [\%] & 0.3550 & 3.1481 \\
& ISE & 0.4660 & 0.5347 \\
& ISE-AB & 3.5746 & 4.0468 \\
\hline ISE-ST & Rise Time [s] & 1.0120 & 1.6997 \\
& Settling Time [s] & 1.8747 & 2.8462 \\
& Overshoot [\%] & 1.2320 & 1.9231 \\
& ISE & 0.4709 & 0.5979 \\
& ISE-ST & 0.6583 & 0.8825 \\
\hline ISE-OS & Rise Time [s] & 1.1723 & 1.5896 \\
& Settling Time [s] & 3.5311 & 5.5525 \\
& Overshoot [\%] & 0 & 0.1948 \\
& ISE & 0.4735 & 0.5724 \\
& ISE-OS & 0.4753 & 0.5735 \\
\hline & & &
\end{tabular}

TABLE IV

PID CONTROL GAINS

\begin{tabular}{cccc}
\hline Objective Function & $\boldsymbol{K}_{\boldsymbol{p}}^{\boldsymbol{\theta}}$ & $\begin{array}{c}\text { Angle } \\
\boldsymbol{K}_{\boldsymbol{i}}^{\boldsymbol{\theta}}\end{array}$ & $\boldsymbol{K}_{\boldsymbol{d}}^{\boldsymbol{\theta}}$ \\
\hline PID (Prasad et al (2014)) & -40 & 0 & -8 \\
ISE & -43.9238 & 1.2625 & -6.1163 \\
ISE-ST & -43.6806 & 0.8948 & -6.2171 \\
ISE-OS & -42.3380 & -1.2595 & -6.1730 \\
ISE-AB & -43.8129 & 0.2949 & -6.0142 \\
\hline Objective Function & \multicolumn{3}{c}{ Position } \\
\hline PID (Prasad et al (2014)) & -1 & $\boldsymbol{K}_{\boldsymbol{i}}^{\boldsymbol{x}}$ & $\boldsymbol{K}_{\boldsymbol{d}}^{\boldsymbol{x}}$ \\
ISE & -2.8623 & -0.0017 & -3.5402 \\
ISE-ST & -2.5071 & -0.0279 & -3.2817 \\
ISE-OS & -1.8106 & 0 & -2.6507 \\
ISE-AB & -2.3795 & 0 & -3.1028 \\
\hline
\end{tabular}

in the control scheme, they were used as additional measures to compare the results of the various PID tuning schemes.

\section{B. Combined Control Schemes}

The performance of the full aggregate control scheme is studied where the PID and the feedback optimization loops are both enabled. The weighting matrices, which are used to find the Riccati solution and to form the objective function of the neural network are, set to $Q=\operatorname{diag}\{1,1,500,250\}$, $R=1, Q^{N N}=\operatorname{diag}\{1,1,50,25\}$, and $R^{N N}=0.016$. The control law calculated using the Riccati approach is given by $K=[-137.7896-25.9783-22.3607-27.5768]$ [20]. The ANN training information are detailed in Table VI. The cart and pendulum position responses as well as the control signal are shown in Figs. 5, 6, and 7 and the results are summarized 
TABLE V

PERFORMANCE OF PID CONTROL UNITS TUNED USING DIFFERENT COST CRITERIA

\begin{tabular}{ccccccc}
\hline Method & Rise time [s] & Settling time [s] & Overshoot [\%] & ISE & $\int_{\mathbf{0}}^{t} U(X(\eta), \boldsymbol{u}(\boldsymbol{\eta})) d \boldsymbol{\eta}$ & $\int_{\mathbf{0}}^{t} \boldsymbol{F}(\boldsymbol{X}(\boldsymbol{\eta}), \boldsymbol{u}(\boldsymbol{\eta})) \boldsymbol{d} \boldsymbol{\eta}$ \\
\hline PID (Prasad et al (2014)) & 4.8914 & 9.6098 & $\mathbf{0}$ & 0.72255 & 800.1996 & 79.5381 \\
PID (ISE) & $\mathbf{0 . 8 9 0 0}$ & 3.3351 & 3.4130 & $\mathbf{0 . 4 5 5 8}$ & 686.3625 & 67.2464 \\
PID (ISE-ST) & 1.0120 & $\mathbf{1 . 8 7 4 7}$ & 1.7828 & 0.4709 & 674.2809 & 66.3176 \\
PID (ISE-OS) & 1.3283 & 3.8942 & $\mathbf{0}$ & 0.5093 & $\mathbf{6 6 2 . 3 5 8 1}$ & $\mathbf{6 5 . 4 6 7 4}$ \\
PID (ISE-AB) & 1.0578 & 1.9593 & 1.2077 & 0.4751 & 665.5093 & 65.5258 \\
\hline
\end{tabular}

in Table VII. Once again, the proposed architecture along with the PID variants outperformed the PID-LQR system suggested in [20]. The improvement in terms of the measures $\int_{0}^{t} U(X(\eta), u(\eta)) \mathrm{d} \eta$ and $\int_{0}^{t} F(X(\eta), u(\eta)) \mathrm{d} \eta$ reached up to $46 \%$ and $47 \%$, respectively. This reveals how the ANN loop was successful in reducing these two measures with respect to the PID control structure (comparing Tables V and VII).

TABLE VI

Parameters of the Neural Network Optimizer

\begin{tabular}{cc}
\hline Parameters & Values \\
\hline$X_{\min }, X_{\max }$ & $\pm[0.175 \mathrm{rad} 0.35 \mathrm{rad} / \mathrm{s} 0.1 \mathrm{~m} 0.2 \mathrm{~m} / \mathrm{s}]$ \\
$u_{\min }, u_{\max }$ & $\pm 5 \mathrm{~N}$ \\
$\Delta X$ & $\left(X_{\max }-X_{\min }\right) / 20$ \\
$\Delta u^{N N}$ & $\left(u_{\max }^{N N}-u_{\min }^{N N}\right) / 20$ \\
Number of hidden layers & 1 \\
Number of hidden neurons & 13 \\
Size of data set & 194,481 samples \\
(Training,Validation,Testing)\% & $(70,15,15) \%$ \\
Training mechanism & Levenberg-Marquardt \\
\hline
\end{tabular}

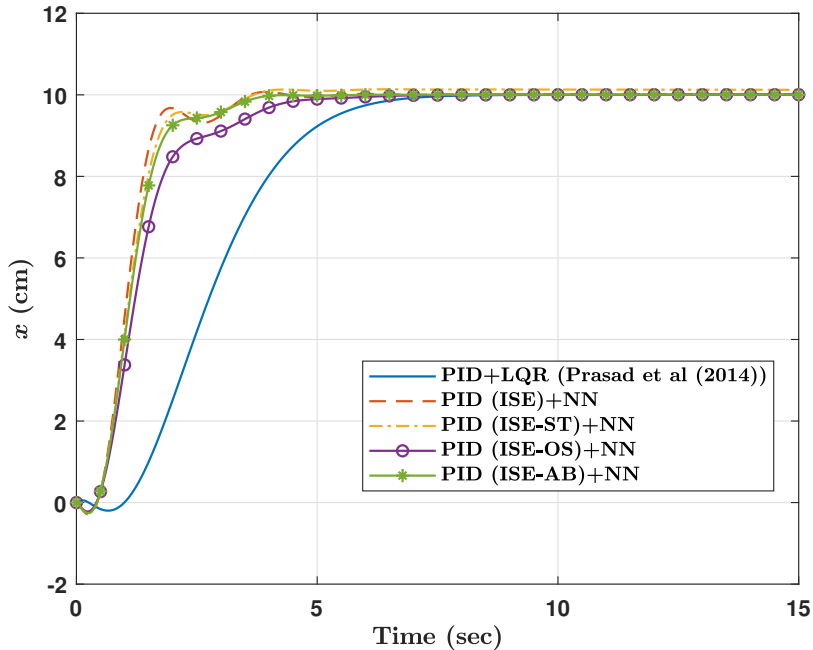

Fig. 5. Cart position $x$ (under aggregate control structures)

The cart reference position $x^{r e f}(t)$ is assumed to follow a square wave command letting the cart slides back and forth between 8 and $12 \mathrm{~cm}$ using the full control system. The cart position responses and the cumulative cart position tracking-error $\int_{0}^{t}\left|e_{x}(\eta)\right| \mathrm{d} \eta$ are displayed in Figs. 8 and 9. The proposed architecture resulted in better transient and steadystate characteristics and better overall dynamic cost than the controller of [20].

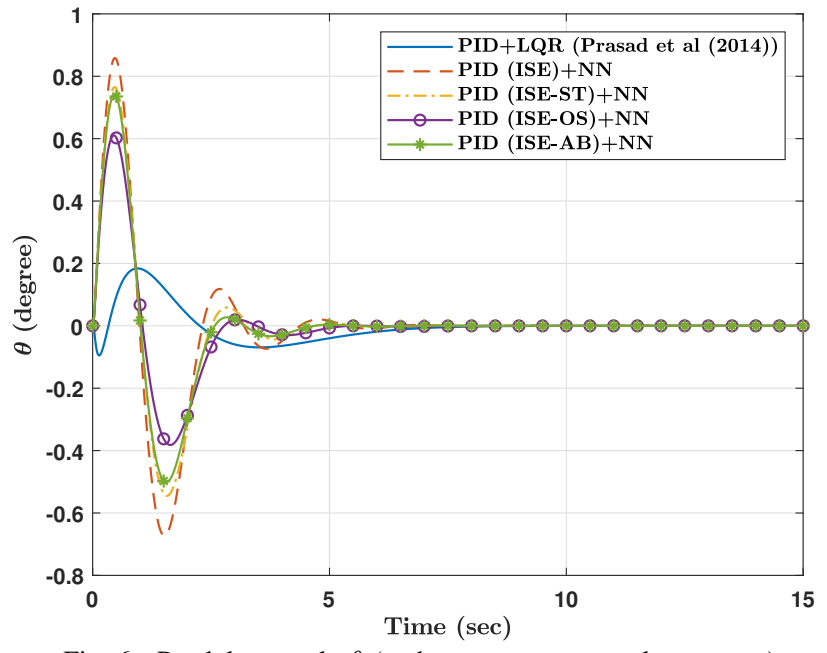

Fig. 6. Pendulum angle $\theta$ (under aggregate control structures)

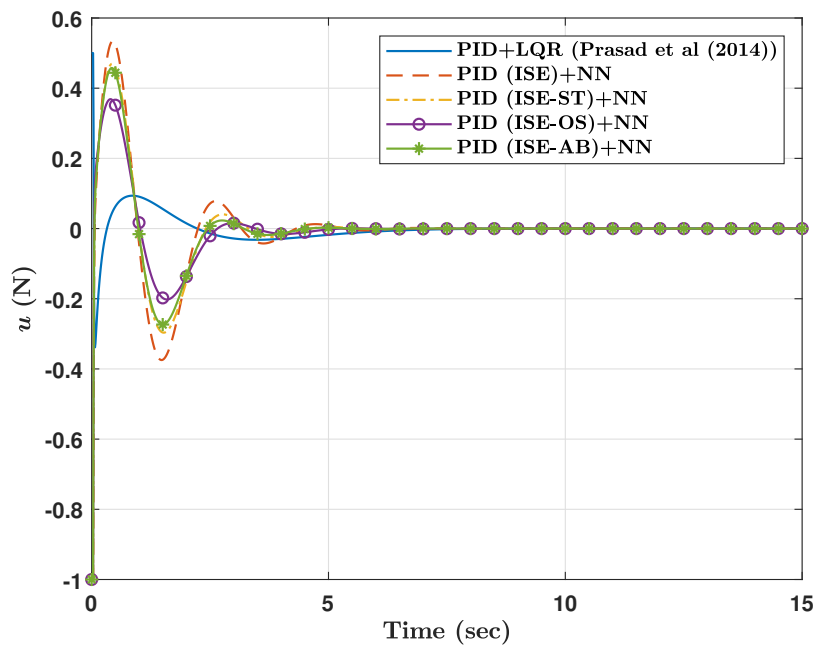

Fig. 7. Control signal $u$ (under aggregate control structures)

\section{CONCLUSION}

This work generalizes a nonlinear threshold accepting heuristic along with ANN optimization tool to control multioutput balance systems. The techniques were applied to a system with two outputs and a PID control unit was applied to track each output. It is demonstrated how various objective functions can be integrated in the NLTA scheme to search for optimized PID gains in order to satisfy certain design criteria pertaining to the transient and steady state characteristics. The results were benchmarked against a control algorithm suggested in the literature. It was outperformed by the proposed mechanisms in all conducted simulations with and without the 
TABLE VII

PERFORMANCE USING COMBINED CONTROL STRUCTURES (PID+LQR VERSUS PID+NN)

\begin{tabular}{ccccccc}
\hline Method & Rise time [s] & Settling time [s] & Overshoot [\%] & ISE & $\int_{\mathbf{0}}^{\boldsymbol{t}} \boldsymbol{U}(\boldsymbol{X}(\boldsymbol{\eta}), \boldsymbol{u}(\boldsymbol{\eta})) \boldsymbol{d} \boldsymbol{\eta}$ & $\int_{\mathbf{0}}^{\boldsymbol{t}} \boldsymbol{F}(\boldsymbol{X}(\boldsymbol{\eta}), \boldsymbol{u}(\boldsymbol{\eta})) \boldsymbol{d} \boldsymbol{\eta}$ \\
\hline PID + LQR (Prasad et al (2014)) & 3.2407 & 6.1969 & $\mathbf{0}$ & 1.1437 & 1207.6 & 120.5957 \\
PID (ISE) + NN & $\mathbf{0 . 9 5 4 6}$ & $\mathbf{3 . 3 2 7 3}$ & 0.6194 & $\mathbf{0 . 4 5 7 6}$ & 672.7276 & 65.8731 \\
PID (ISE-ST) + NN & 1.1275 & 3.5240 & 0.1413 & 0.4733 & 661.1241 & 65.0051 \\
PID (ISE-OS) + NN & 2.0745 & 4.3102 & $\mathbf{0}$ & 0.5199 & 659.5133 & 65.1875 \\
PID (ISE-AB) + NN & 1.2055 & 3.4214 & 0.0103 & 0.4790 & $\mathbf{6 5 4 . 4 6 9 2}$ & $\mathbf{6 4 . 4 2 3 1}$ \\
\hline
\end{tabular}

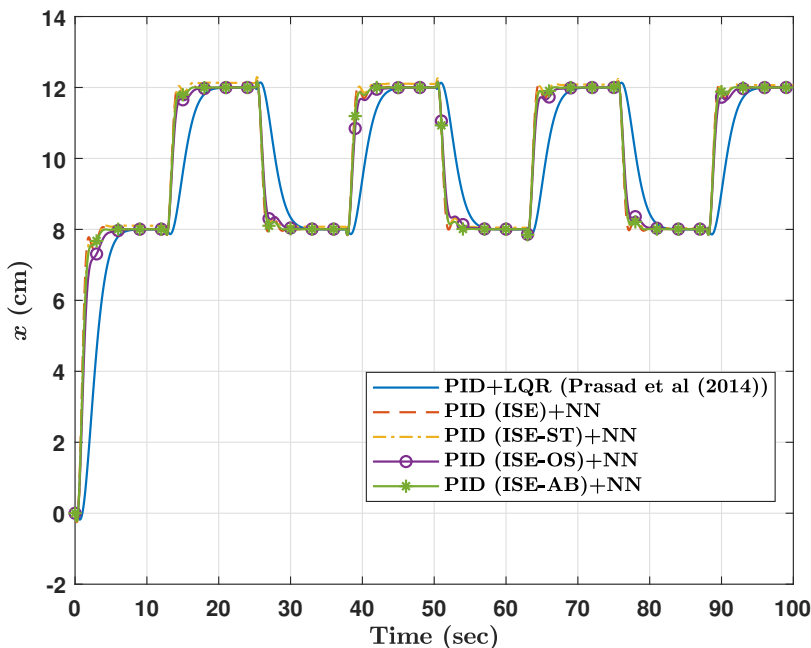

Fig. 8. Cart position $x$ (with square wave reference under aggregate control structures)

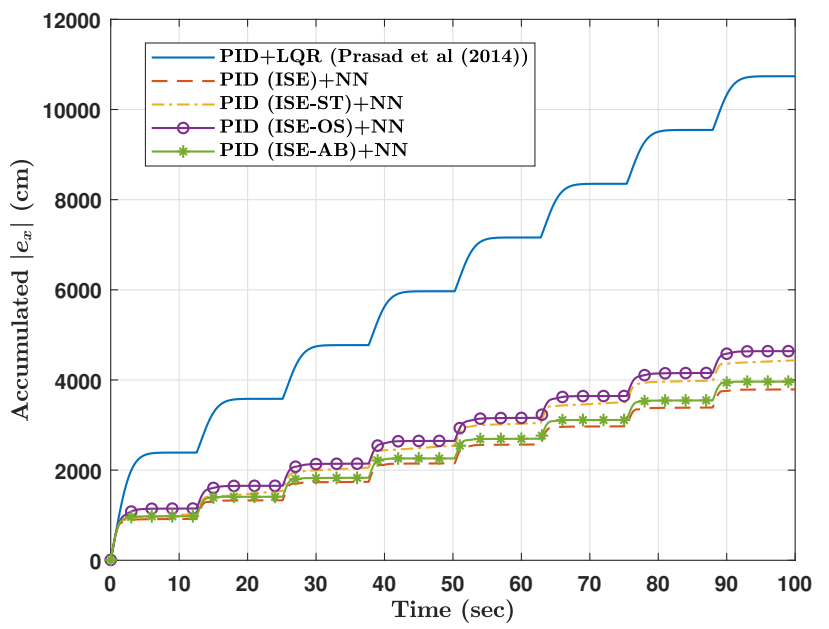

Fig. 9. Cumulative cart position error (with square wave reference under aggregate control structures)

ANN optimization loop. The study showed an improvement in the optimization cost measures of up to $47 \%$.

\section{REFERENCES}

[1] S. Jung and S. S. Kim, "Control Experiment of a Wheel-Driven Mobile Inverted Pendulum Using Neural Network," IEEE Transactions on Control Systems Technology, vol. 16, no. 2, pp. 297-303, Mar. 2008.

[2] M. I. H. Nour, J. Ooi, and K. Y. Chan, "Fuzzy logic control vs. conventional PID control of an inverted pendulum robot," in 2007 International Conference on Intelligent and Advanced Systems. Kuala Lumpur: IEEE, Nov. 2007, pp. 209-214.

[3] A. K. Yadav, P. Gaur, A. P. Mittal, and M. Anzar, "Comparative analysis of various control techniques for inverted pendulum," in India
International Conference on Power Electronics 2010 (IICPE2010). New Delhi, India: IEEE, Jan. 2011, pp. 1-6.

[4] S. Bennett, "A brief history of automatic control," IEEE Control Systems Magazine, vol. 16, no. 3, pp. 17-25, Jun. 1996.

[5] V. Parra-Vega, S. Arimoto, Yun-Hui Liu, G. Hirzinger, and P. Akella, "Dynamic sliding PID control for tracking of robot manipulators: theory and experiments," IEEE Transactions on Robotics and Automation, vol. 19, no. 6, pp. 967-976, Dec. 2003.

[6] G. Szafranski and R. Czyba, "Different approaches of pid control uav type quadrotor," The International Micro Air Vehicles Conference, pp. 70-75, 012011.

[7] Zulfatman and M. F. Rahmat, "Application of self-tuning fuzzy PID controller on industrial hydraulic actuator using system identification approach," International Journal on Smart Sensing and Intelligent Systems, vol. 2, no. 2, pp. 246-261, 2009.

[8] W. W. Shein, Y. Tan, and A. O. Lim, "PID Controller for Temperature Control with Multiple Actuators in Cyber-Physical Home System," in 2012 15th International Conference on Network-Based Information Systems. Melbourne, Australia: IEEE, Sep. 2012, pp. 423-428.

[9] N. Nahas and M. Nourelfath, "Nonlinear threshold accepting metaheuristic for combinatorial optimisation problems," International Journal of Metaheuristics, vol. 3, no. 4, pp. 265-290, Jan. 2014.

[10] N. Wang, M. Abouheaf, W. Gueaieb, and N. Nahas, "Model-free optimized tracking control heuristic," Robotics, vol. 9, no. 3, p. 49, Jun 2020. [Online]. Available: http://dx.doi.org/10.3390/robotics9030049

[11] N. Nahas, M. Noomane Darghouth, and M. Abouheaf, "A non-linearthreshold-accepting function based algorithm for the solution of economic dispatch problem," RAIRO - Operations Research, Apr. 2019.

[12] N. Nahas, M. N. Darghouth, A. Q. Kara, and M. Nourelfath, "Nonlinear threshold algorithm based solution for the redundancy allocation problem considering multiple redundancy strategies," Journal of Quality in Maintenance Engineering, vol. 25, no. 3, pp. 397-411, Aug. 2019.

[13] N. Nahas, M. Abouheaf, A. Sharaf, and W. Gueaieb, "A Self-Adjusting Adaptive AVR-LFC Scheme for Synchronous Generators," IEEE Transactions on Power Systems, vol. 34, no. 6, pp. 5073-5075, Nov. 2019.

[14] D. Park, M. El-Sharkawi, R. Marks, L. Atlas, and M. Damborg, "Electric load forecasting using an artificial neural network," IEEE Transactions on Power Systems, vol. 6, no. 2, pp. 442-449, May 1991.

[15] F.-C. Chen and H. K. Khalil, "Adaptive control of nonlinear systems using neural networks," International Journal of Control, vol. 55, no. 6 , pp. 1299-1317, Jun. 1992.

[16] B. S. Kim and A. J. Calise, "Nonlinear flight control using neural networks," Journal of Guidance, Control, and Dynamics, vol. 20, no. 1, pp. 26-33, 1997.

[17] A. Chakrabarty, C. Danielson, S. Di Cairano, and A. Raghunathan, "Active learning for estimating reachable sets for systems with unknown dynamics," IEEE Transactions on Cybernetics, pp. 1-12, 2020.

[18] C. Mu, K. Wang, and T. Qiu, "Dynamic event-triggering neural learning control for partially unknown nonlinear systems." IEEE transactions on cybernetics, vol. PP, 2020.

[19] M. Abouheaf and W. Gueaieb, "Neurofuzzy Reinforcement Learning Control Schemes for Optimized Dynamical Performance," in 2019 IEEE International Symposium on Robotic and Sensors Environments (ROSE), Jun. 2019, pp. 1-7.

[20] L. B. Prasad, B. Tyagi, and H. O. Gupta, "Optimal Control of Nonlinear Inverted Pendulum System Using PID Controller and LQR: Performance Analysis Without and With Disturbance Input," International Journal of Automation and Computing, vol. 11, no. 6, pp. 661-670, Dec. 2014.

[21] F. L. Lewis, D. Vrabie, and V. L. Syrmos, Optimal Control. John Wiley \& Sons, Mar. 2012. 